\title{
Adaptive Correction Forecasting Approach for Urban Traffic Flow Based on Fuzzy $c$-Mean Clustering and Advanced Neural Network
}

\author{
He Huang, ${ }^{1}$ Qifeng Tang, ${ }^{2}$ and Zhen Liu ${ }^{1}$ \\ ${ }^{1}$ Shanghai Urban-Rural Construction and Transportation Development Institute, Shanghai 300032, China \\ ${ }^{2}$ Department of Automation, Shanghai Jiao Tong University, Key Laboratory of System Control and Information Processing, \\ Ministry of Education, Shanghai 200240, China
}

Correspondence should be addressed to Qifeng Tang; tqf001@qq.com

Received 5 July 2013; Revised 8 October 2013; Accepted 9 October 2013

Academic Editor: Baocang Ding

Copyright (c) 2013 He Huang et al. This is an open access article distributed under the Creative Commons Attribution License, which permits unrestricted use, distribution, and reproduction in any medium, provided the original work is properly cited.

Forecasting of urban traffic flow is important to intelligent transportation system (ITS) developments and implementations. The precise forecasting of traffic flow will be pretty helpful to relax road traffic congestion. The accuracy of traditional single model without correction mechanism is poor. Summarizing the existing prediction models and considering the characteristics of the traffic itself, a traffic flow prediction model based on fuzzy $c$-mean clustering method (FCM) and advanced neural network (NN) was proposed. FCM can improve the prediction accuracy and robustness of the model, while advanced NN can optimize the generalization ability of the model. Besides these, the output value of the model is calibrated by the correction mechanism. The experimental results show that the proposed method has better prediction accuracy and robustness than the other models.

\section{Introduction}

Real-time forecasting of traffic flow is an important issue in advanced traffic management [1]. The traffic simulation is correspondingly needed to make these forecasting models reliable way, which aim to influence travel behavior, reduce traffic congestion, improve mobility, and enhance air quality. Traffic forecasting models can be used to provide urban traffic control centers with an automated tool for anticipating the congestion that may arise on road facilities and its expected duration [2].

The urban traffic flow forecasting models rely on historical and current flow data. The problem of traffic flow forecasting belongs to a standard time series prediction task and the purpose is to fetch the function which can relates future values of traffic flow to previous and current measurement of traffic flow [3]. A variety of forecasting techniques has been applied to forecast the urban traffic flow. In [4], DanechPajouh and Aron designed a layered statistical method with a mathematical clustering technique to group the traffic flow data and a separately tuned linear regression model for each cluster. The ARIMA model, initially developed by Kim et al., is one of the most popular approaches in traffic flow forecasting [5-7]. However, the limitation of ARIMA models is that their natural tendency, concentrated on the mean values of the past series data, seems unable to capture the rapid varying process changes underlying of traffic flow. The artificial neural network (ANN) is widely applied in traffic flow forecasting. Yin et al. [8] developed a fuzzy-neural model (FNM) to predict traffic flow in an urban street network. The empirical results showed that the FNM model provides more accurate forecasting results than the BPNN model. These researches are committed to improve the performance of the algorithms. However, there are many factors which can affect the traffic flow, the traditional single model can hardly improve the prediction accuracy and no online correction mechanism was considered. This motivates the paper.

In this paper, the traffic flow forecasting model has 3 techniques: first, the input data of the model is divided into several categories according to FCM, and different categories have different model. Second, a training model based on a well-defined part-connected neural network (NN) was proposed and the cooperative quantum-particle swarm evolutionary algorithm (CQGAPSO) is used to train the 
model. Last, the error between predicted value and real value is used to compensate the output of the model. These methods can improve the accuracy and generalization of the forecasting model can also overcome the model mismatch.

This paper is organized as follows. The forecasting methodology is introduced in Section 2. Cases of studying of urban traffic flow forecasting are given in Section 3. Conclusions are finally made in Section 4.

\section{Forecasting Methodology}

2.1. The Framework of the Proposed Method. According to the change rule of traffic flow time series, there is an essential linkage between the future and the previous flow [9]. Thus, the previous traffic flow value can be used to forecast the future flow. Set $f(t)$ as the traffic flow at time $t, f(t-1)$ as the value at time $t-1$. In this paper, $f(t), f(t-1), \ldots$, and $f(t-s)$ are the input values of the model at time $t$ and $f(t+p)$ is the predicted value at time $t+p$. The input values are denoted as $x(i)$ and the predicted value is denoted $y(i)$. The traffic flow forecasting model is made to build the relationship between $y(i)$ and $x(i)$. Therefore, once the relationship is obtained, the model can be used to predict the future traffic flow based on the real-time measured data in practice.

In the previous studies, the single prediction model mentioned above was adopted to forecast the urban traffic flow. However, it is not universally applicable for all the traffic scenarios. Since the urban traffic system is an unstable system, which exhibits significant variation in different periods, it is necessary to establish different prediction models to forecast the future traffic state accurately. According to the measured data from the float car, Guo et al. [10] analyzed the degree of traffic congestion on different days in a week. The results showed that the traffic congestion of Monday is more serious than the other days, especially in the morning peak hour, and the most serious traffic congestion of evening peak hour occurred in Friday. Moreover, the degree of traffic congestion during commuting time on the weekend is less than the degree on weekdays. It can be concluded that by observing the traffic flow data, the travel modes and travel demand are different on each day of a week, and the data characteristic of the same day for every week is similar. Therefore, in order to improve the accuracy of prediction for traffic flow or travel speed on the road, it is necessary to classify the traffic flow pattern and apply a suitable model to forecast each pattern. This classification would guarantee that each prediction model has a good performance in a particular period. As urban traffic flow system is a complicated process influenced by many factors, it is believed that using the multimodel method to predict the traffic flow is appropriate.

From the analysis made above, in this paper, for the sake of modeling, the historical traffic data should be divided into seven classes corresponding to each day of a week. Besides, considering the widely variation of traffic flow from morning to night, especially in the rush hour, using a single model to describe a complex nonlinear object usually results in low accuracy and poor generalization. So we use FCM to process the data and choose the reasonable clustering number by

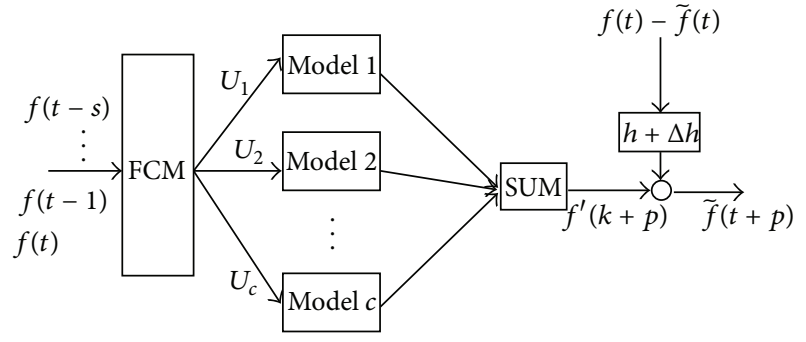

FIGURE 1: The framework of the proposed method.

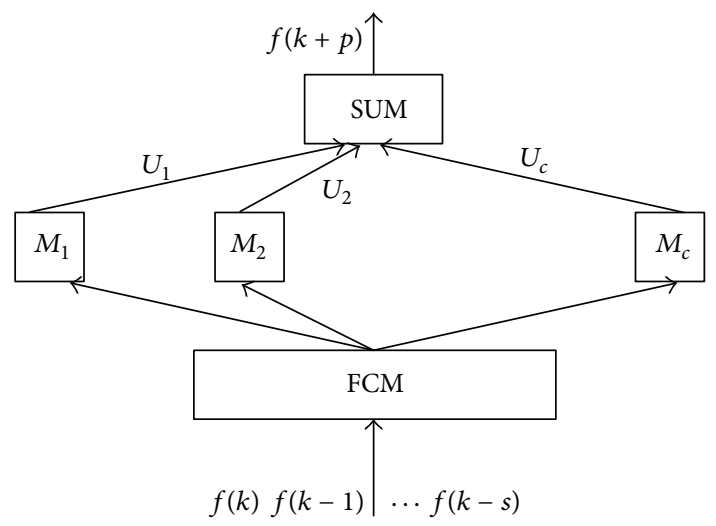

FIgURE 2: The architecture of FCM.

the experiments and use the approach based on multipleinput-single-output three-layer feedforward neural network with switches to model each cluster. Meanwhile, in order to overcome the model mismatch, the adaptive correction mechanism is added to our approach. The framework of the proposed method is illustrated in Figure 1.

2.2. Fuzzy c-Means Clustering. The model of forecasting traffic flow is a multiinput single-output system; the training sample set can be expressed as $D=\left\{f_{i}(t+p),\left[f_{i}(t) f_{i}(t-\right.\right.$ $\left.\left.1), \ldots, f_{i}(t-s)\right] \mid s=1,2 \ldots m, i=1,2, \ldots, n\right\}$. Here, $n$ is the sample number of training set, $m$ is the number of input variables; $\left[f_{i}(t) f_{i}(t-1), \ldots, f_{i}(t-s)\right]$ denotes the $i$ th input vector. Suppose $D$ is divided into $c$ clusters $\left\{D_{1}, D_{2}, \ldots D_{c}\right\}$; thus, $c$ submodels $\left\{M_{1}, M_{2}, \ldots M_{c}\right\}$ should be built for each $D_{i}$, and the result of the FCM can be expressed as membership matrix $U=\left[u_{i j}\right]_{i=1,2 \ldots, \ldots, j=1,2 \ldots n}=$ $\left\{U_{i} \mid i=1,2 \ldots c\right\} . u_{i j}$ denotes the degree of the element $x_{j}$ in training sample set belonging to the $i$ th cluster. The value of $u_{i j}$ is between 0 and 1 . The architecture of FCM method is shown in Figure 2 [11, 12].

Clustering number $c$ is a very important parameter. Here, we do experiments to choose the appropriate clustering number $c$. Let $c$ increase from 2 to a constant. Then, make models separately based on FCM and calculate the mean square error and the maximum error according to (1). Last, we can obtain the best clustering number $c$.

Consider

$$
\begin{aligned}
& \text { MSE }=\left(\frac{1}{n_{1}} \sum_{i=1}^{n_{1}}\left(y_{i}-\widetilde{y_{i}}\right)^{2}\right)^{0.5}, \\
& \text { MAXE }=\max _{i=1}^{n_{1}}\left(\left|y_{i}-\widetilde{y_{i}}\right|\right) .
\end{aligned}
$$


TABLE 1: The result of the FCM.

\begin{tabular}{|c|c|c|c|c|c|c|}
\hline \multirow{2}{*}{ Clustering number $c$} & \multicolumn{2}{|c|}{ Monday 1} & \multicolumn{2}{|c|}{ Wednesday 1} & \multicolumn{2}{|c|}{ Sunday 1} \\
\hline & MSE & MAXE & MSE & MAXE & MSE & MAXE \\
\hline 1 & 14.0515 & 57.5568 & 14.2659 & 61.2561 & 14.1235 & 57.3078 \\
\hline 2 & 13.3489 & 51.3476 & 14.0024 & 53.1487 & 13.2149 & 51.0947 \\
\hline 3 & 10.0456 & 43.1834 & 13.4820 & 48.4621 & 9.8952 & 42.1001 \\
\hline 4 & 11.8439 & 44.9576 & 11.2106 & 45.2548 & 11.7541 & 44.8259 \\
\hline 5 & 13.2563 & 47.5963 & 13.5279 & 49.3247 & 13.1589 & 46.2985 \\
\hline \multirow{2}{*}{ Clustering number $c$} & \multicolumn{2}{|c|}{ Monday 2} & \multicolumn{2}{|c|}{ Wednesday 2} & \multicolumn{2}{|c|}{ Sunday 2} \\
\hline & MSE & MAXE & MSE & MAXE & MSE & MAXE \\
\hline 1 & 15.1125 & 60.9547 & 14.3762 & 59.0143 & 14.4321 & 60.6465 \\
\hline 2 & 14.5876 & 54.2154 & 14.1144 & 59.9821 & 14.2547 & 61.6435 \\
\hline 3 & 11.5248 & 40.1257 & 13.5520 & 50.7234 & 13.6464 & 50.9542 \\
\hline 4 & 12.5487 & 42.2037 & 11.3017 & 47.0984 & 12.3014 & 48.2549 \\
\hline 5 & 13.6587 & 44.1023 & 13.6975 & 51.2459 & 13.4164 & 50.8216 \\
\hline
\end{tabular}

2.3. The Forecasting Model Based on Neural Network with Switches. In the architecture of FCM method, each model needs a modeling tool. NN, SVM, and Kalman filtering are always used to forecast the traffic flow. Here, we adopt an advanced $\mathrm{NN}$, the multiple-input-single-output three-layer feedforward neural network with switches was proposed and well defined in [13]. A multiple-input-single-output (MISO) three-layer feedforward neural work with switches is shown in Figure 3.

Various methods were proposed to train the $\mathrm{NN}$ with switches [13-15]. In those methods, the population was partitioned to parameters and structure population. The parameters population was composed of the weight of the links, while the structure population was composed of the link switches. This model could eliminate some ill effects of approximation ability caused by redundant structure of $\mathrm{NN}$.

2.4. The Adaptive Correction Mechanism. The traffic flow is the measurable variable, and the real-time data is used to predict the future traffic flow [16]. For example, at current time $t$, we can obtain the real value $f(t)$ from the sensors and the predicted value $\widetilde{f}(t)$ by forecasting the model. Here is an error $e=f(t)-\tilde{f}(t)$ because of the model mismatch. At time $k$, the model should forecast the traffic flow at time $k+p$; the error can be used to compensate the initial predicted value $f^{\prime}(t+p)$ according to (2). $h$ is the offline correction coefficient.

Consider

$$
\tilde{f}(t+p)=f^{\prime}(t+p)+h \cdot e .
$$

The training set $D$ can be used to fetch $h$; to fetch $h$ is to find the relationship between $f(t)-\tilde{f}(t)$ and $f(t+p)-\widetilde{f}(t+p)$, and here, $t=1,2 \ldots m, m$ is the sample number of training set. $h$ can be calculated by least square method (SLM).

When the model is forecasting the traffic flow online, the correction coefficient $h$ should be refreshed in real time. For example, at current time $t$, we can calculate $\Delta h$ using a small piece of historical data to obtain the relationship between $f(t-i-p)-\tilde{f}(t-i-p)$ and $f(t-i)-\tilde{f}(t-i)$. Here, $i$ is a small positive integer. The online correction coefficient $\Delta h$ can be obtained by SLM and (2) should be modified.

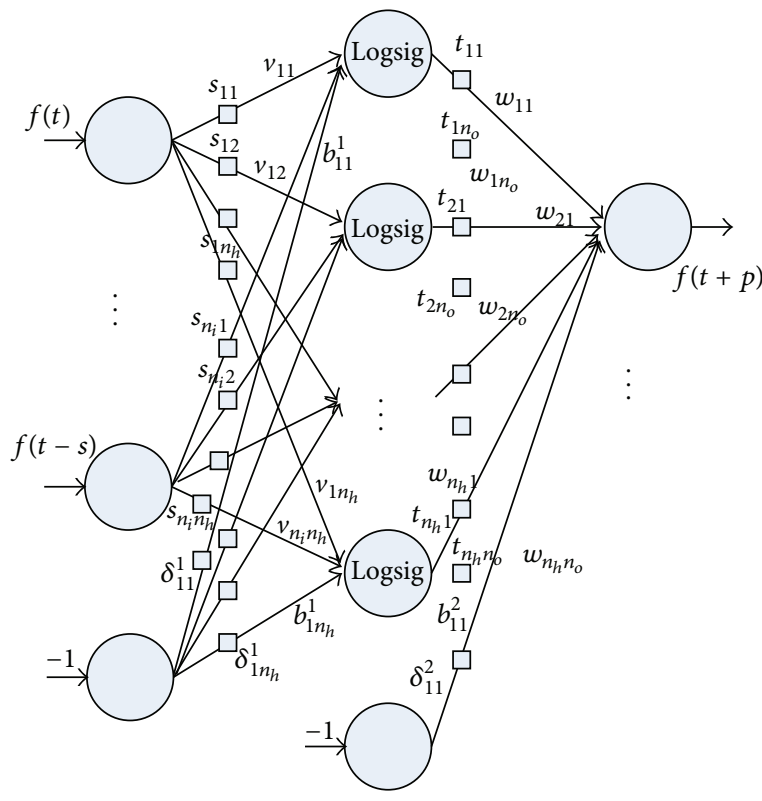

$\square$ Switches

FIGURE 3: The structure of three-layer feedforward NN with switches.

Consider

$$
\tilde{f}(t+p)=f^{\prime}(k+p)+(h+\Delta h) \cdot e .
$$

\section{Experimental Results}

In order to explain the effectiveness of the proposed method, we choose the data gathered from Shanghai north-south highway including from August to October. The historical data on August and September is used to build the training set, while the data on October is used to build the testing set. There is a large difference of traffic flow every day in a week, thus we build different models for every day. Here, we use the first two Monday, Wednesday and Sunday on October to verify the proposed model. 
TABLE 2: The result of adding the correction mechanism.

\begin{tabular}{|c|c|c|c|c|c|c|}
\hline \multirow{2}{*}{$h+\Delta h$} & \multicolumn{2}{|c|}{ Monday 1} & \multicolumn{2}{|c|}{ Wednesday 1} & \multicolumn{2}{|c|}{ Sunday 1} \\
\hline & MSE & MAXE & MSE & MAXE & MSE & MAXE \\
\hline$h+\Delta h=0$ & 10.0456 & 43.1843 & 11.2106 & 45.2548 & 9.8952 & 42.1001 \\
\hline$-0.1 \leq \Delta h \leq 0.1$ & 9.9758 & 42.1285 & 10.2654 & 44.1657 & 9.7561 & 40.6548 \\
\hline$-0.2 \leq \Delta h \leq 0.2$ & 15.2648 & 59.2154 & 16.2299 & 61.2147 & 14.3215 & 57.6519 \\
\hline$-0.3 \leq \Delta h \leq 0.3$ & 99.2154 & 70.2165 & 105.2647 & 85.2594 & 99.0147 & 69.2589 \\
\hline \multirow{2}{*}{$h+\Delta h$} & \multicolumn{2}{|c|}{ Monday 2} & \multicolumn{2}{|c|}{ Wednesday 2} & \multicolumn{2}{|c|}{ Sunday 2} \\
\hline & MSE & MAXE & MSE & MAXE & MSE & MAXE \\
\hline$h+\Delta h=0$ & 11.5248 & 40.1257 & 11.3017 & 47.0984 & 12.3014 & 48.2549 \\
\hline$-0.1 \leq \Delta h \leq 0.1$ & 10.4529 & 38.2489 & 10.3594 & 46.2813 & 11.2497 & 47.9523 \\
\hline$-0.2 \leq \Delta h \leq 0.2$ & 16.5489 & 58.2146 & 17.2016 & 64.0525 & 16.2018 & 58.2687 \\
\hline$-0.3 \leq \Delta h \leq 0.3$ & 103.4269 & 88.2159 & 106.2184 & 86.3468 & 101.4512 & 98.1264 \\
\hline
\end{tabular}

TABLE 3: The comparison of 3 different models.

\begin{tabular}{|c|c|c|c|c|c|c|}
\hline & \multicolumn{2}{|c|}{ Monday 1} & \multicolumn{2}{|c|}{ Wednesday 1} & \multicolumn{2}{|c|}{ Sunday 1} \\
\hline & MSE & MAXE & MSE & MAXE & MSE & MAXE \\
\hline Model (a) & 13.4956 & 46.7109 & 14.3495 & 48.3459 & 12.2304 & 46.0239 \\
\hline Model (b) & 10.0456 & 43.1834 & 11.2106 & 45.2548 & 9.8952 & 42.1001 \\
\hline \multirow[t]{3}{*}{ Model (c) } & 9.9758 & 42.1285 & 10.2654 & 44.1657 & 9.7561 & 40.6548 \\
\hline & \multicolumn{2}{|c|}{ Monday 2} & \multicolumn{2}{|c|}{ Wednesday 2} & \multicolumn{2}{|c|}{ Sunday 2} \\
\hline & MSE & MAXE & MSE & MAXE & MSE & MAXE \\
\hline Model (a) & 15.2430 & 49.4545 & 14.4506 & 51.4539 & 16.1356 & 55.2341 \\
\hline Model (b) & 11.5248 & 40.1257 & 11.3017 & 47.0984 & 12.3014 & 48.2549 \\
\hline Model (c) & 10.4529 & 38.2489 & 10.3594 & 46.2813 & 11.2497 & 47.9523 \\
\hline
\end{tabular}

The number of training sample is 2800 and the testing sample number is 650 . There is 2 minutes between each data. Based on the experience, we choose 3 as the dimension of input data. On request, we should predict the traffic flow after 10 minutes. Thus the width of the prediction $p$ is 5 . We totally do 3 experiments: (1) the traditional single model; (2) the multimodels based on FCM; (3) the multimodels based on FCM and adaptive correction mechanism.

First, all the data should be filtered before modeling and NN with switches is used as the modeling tool. Then we should determine the Clustering number $c$ by FCM, "CQGAPSO" algorithm is used to train the NN model and the parameter of "CQGAPSO" algorithm is given in [17]. The hidden nodes number is 6 . The training accuracy is $1 \times 10^{-5}$ and the iteration times of training the $\mathrm{NN}$ are 2000. The experiments are implemented for 50 times. Table 1 gives the result of FCM.

Form Table 1, we can find MSE and MAXE get better after an initial increase in growth of clustering number $c$. However, if $c$ continues to grow, MSE and MAXE will get worse. That is because with the increasing of the clustering number, the generalization ability of the model gets poorer. The best clustering number $c$ is at the turning point. Then the model should be added the adaptive correction mechanism. In order to obtain an appropriate correction coefficient, $h$ is a fixed number which is calculated offline while $\Delta h$ is a changed number which calculated online and we should limit the scope of $\Delta h$. Table 2 gives the result of adding the adaptive correction mechanism. From Table 2, we can find if the adaptive correction mechanism parameter value is $-0.1 \leq$ $\Delta h \leq 0.1$, MSE and MAXE is the best. If the scope of $\Delta h$ is very wide, MSE and MAXE will get worse because the compensation value is too large. Table 3 gives the comparison of every approach. Model (a) is the traditional single model, model (b) is the model (a) with FCM, model (c) is the model (b) with the correction mechanism. We can find the reasonable clustering number $c$ and correction mechanism can improve the forecasting ability.

The Comparison of every approach is illustrated in Figure 3. Figure 4(a) is the traditional single model, Figure 4(b) is the model with FCM, Figure 4(c) is the model with FCM and the correction mechanism. In Figure 4(a), the predictive curve is smooth and cannot track exactly especially at the peak value because the approximation capability of the traditional single model is limited. In Figure 4(b), we can get some submodels by FCM and multimodel can improve the forecasting ability. Without the correction mechanism, the model error cannot be corrected in real time. In Figure 4(c), we use the correction mechanism and it compensates the initial forecasting value with the model error value. From Table 3 and Figure 4, we can find that the predictive accuracy is better than model (a) and (b).

\section{Conclusions}

Aiming at solving the problem of forecasting urban traffic flow, this paper proposes a forecasting model by the use of FCM and correction mechanism. The experimental results 

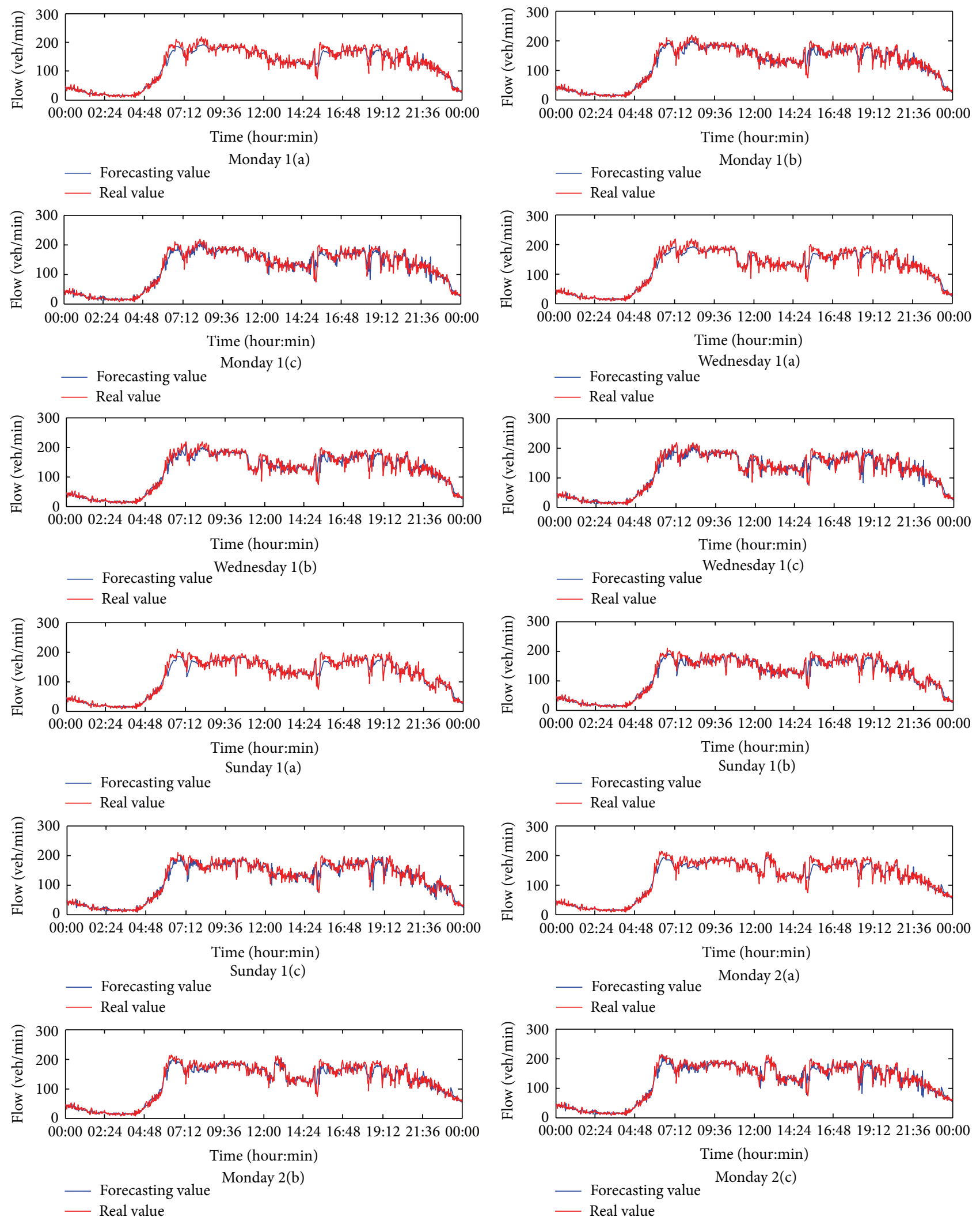

Figure 4: Continued. 

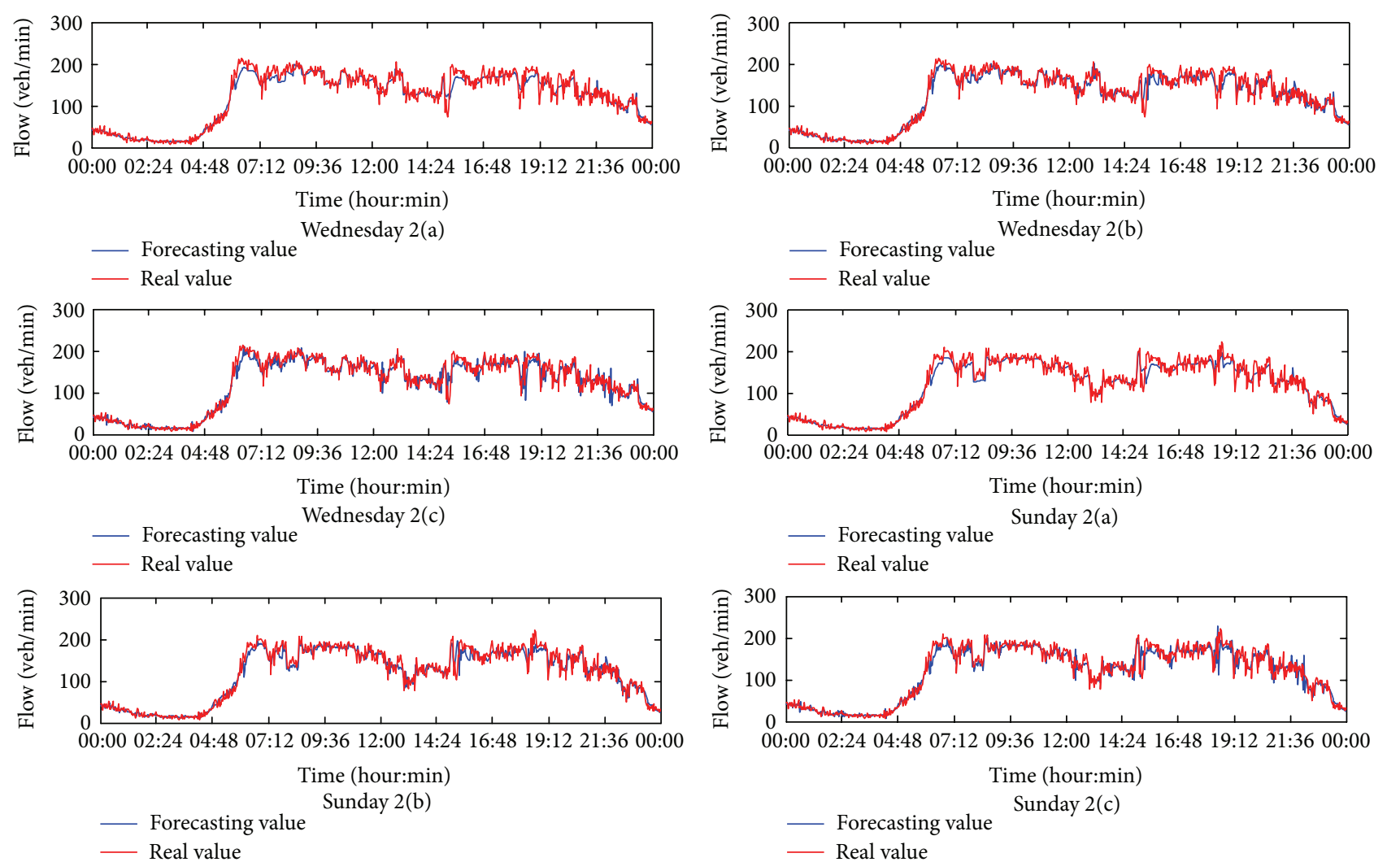

FIGURE 4: the result of forecasting the traffic flow.

indicate that the proposed method can perform better than other methods and show the application prospect.

\section{Acknowledgments}

This paper is supported by the National Science Foundation of China (Grant nos. 71361130012, 61221003), the Specialized Research Fund for the Doctoral Program of Higher Education (Grant no. 20120073110017), and the State Key Laboratory of Synthetical Automation for Process Industries.

\section{References}

[1] M.-W. Li, W.-C. Hong, and H.-G. Kang, "Urban traffic flow forecasting using Gauss-SVR with cat mapping, cloud model and PSO hybrid algorithm," Neurocomputing, vol. 99, no. 1, pp. 230-240, 2013.

[2] T. Pohlmann and B. Friedrich, "A combined method to forecast and estimate traffic demand in urban networks," Transportation Research C, vol. 31, pp. 131-144, 2013.

[3] J. Abdi, B. Moshiri, B. Abdulhai, and A. K. Sedigh, "Forecasting of short-term traffic-flow based on improved neurofuzzy models via emotional temporal difference learning algorithm," Engineering Applications of Artificial Intelligence, vol. 25, no. 5, pp. 1022-1042, 2012.

[4] M. Danech-Pajouh and M. Aron, "Athena: a method for shortterm inter-urban motorway traffic forecasting," Recherche Transports Sécurité, vol. 6, pp. 11-16, 1991.

[5] H.-W. Kim, J.-H. Lee, Y.-H. Choi, Y.-U. Chung, and H. Lee, "Dynamic bandwidth provisioning using ARIMA-based traffic forecasting for Mobile WiMAX," Computer Communications, vol. 34, no. 1, pp. 99-106, 2011.

[6] G. E. P. Box and G. M. Jenkins, Time Series Analysis: Forecasting and Control, Wiley, 1976.

[7] Y. Kamarianakis and P. Prastacos, "Space-time modeling of traffic flow," Computers and Geosciences, vol. 31, no. 2, pp. 119-133, 2005.

[8] H. Yin, S. C. Wong, J. Xu, and C. K. Wong, "Urban traffic flow prediction using a fuzzy-neural approach," Transportation Research Part C, vol. 10, no. 2, pp. 85-98, 2002.

[9] M. Castro-Neto, Y.-S. Jeong, M.-K. Jeong, and L. D. Han, "Online-SVR for short-term traffic flow prediction under typical and atypical traffic conditions," Expert Systems with Applications, vol. 36, no. 3, pp. 6164-6173, 2009.

[10] J. Guo, M. Liu, L. Yu, J. Guan, S. Guo, and X. Zhang, "Development and applications of macroscopic measurement of traffic congestion in Beijing," in Proceedings of the 7th China Annual Conference on Intelligent Transportation Systems, Beijing, China, 2007.

[11] J. C. Bezdek, R. Ehrlich, and W. Full, "FCM: the fuzzy c-means clustering algorithm," Computers and Geosciences, vol. 10, no. 23, pp. 191-203, 1984.

[12] Z. Liu and Q. Liu, "Studying cost-sensitive learning for multiclass imbalance in Internet traffic classification," The Journal of China Universities of Posts and Telecommunications, vol. 19, no. 6, pp. 63-72, 2012.

[13] H. K. Lam, S. H. Ling, F. H. F. Leung, and P. K. S. Tam, “Tuning of the structure and parameters of neural network using an improved genetic algorithm," in Proceedings of the 27th Annual 
Conference of the IEEE Industrial Electronics Society (IECON '2001), pp. 25-30, December 2001.

[14] J.-T. Tsai, J.-H. Chou, and T.-K. Liu, "Tuning the structure and parameters of a neural network by using hybrid Taguchi-genetic algorithm," IEEE Transactions on Neural Networks, vol. 17, no. 1, pp. 69-80, 2006.

[15] G. Yu, G. Li, Y. Bai, and X. Jin, "Tuning of the structure and parameters of wavelet neural network using improved chaotic PSO," in Proceedings of the 26th Chinese Control Conference (CCC '07), pp. 228-232, July 2007.

[16] Q. Tang, D. Li, Y. Xi, and D. Yin, "Soft-sensing design based on semiclosed-loop framework," Chinese Journal of Chemical Engineering, vol. 20, no. 6, pp. 1213-1218, 2012.

[17] Q. F. Tang, The cooperative quantum-particle swarm algorithm and its application in the energy utilization optimization of the steam network [M.E. thesis], East China University of Science\&Technology, China, 2011, (Chinese). 


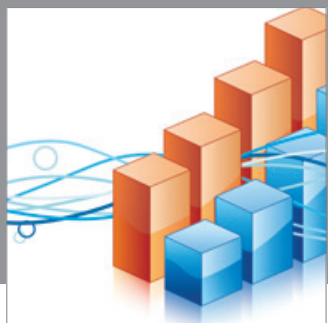

Advances in

Operations Research

mansans

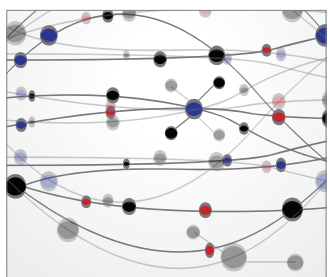

The Scientific World Journal
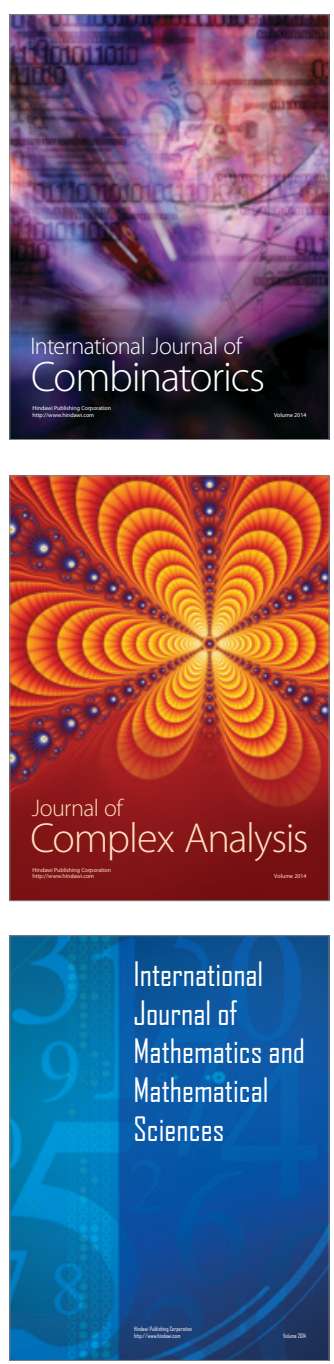
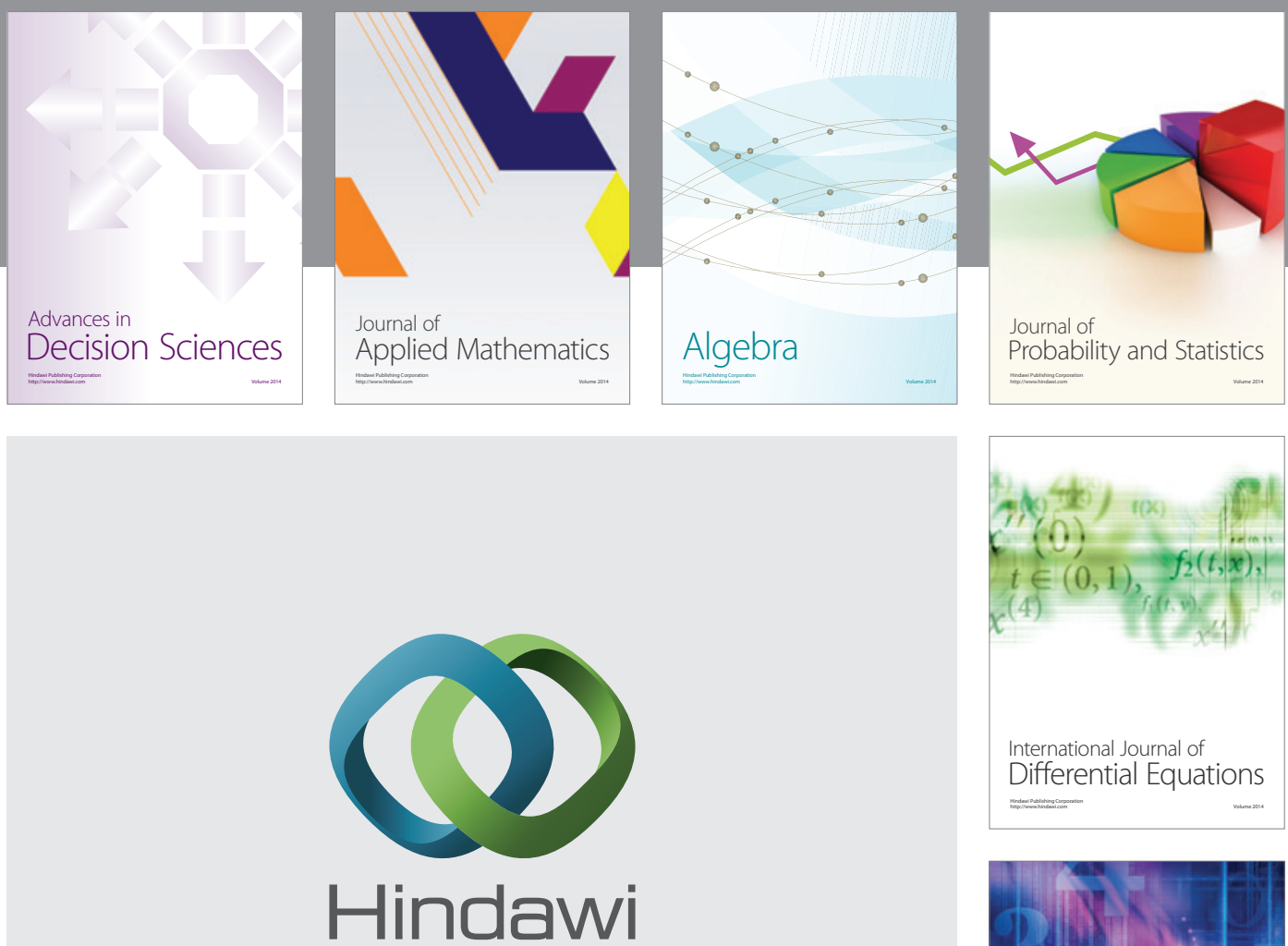

Submit your manuscripts at http://www.hindawi.com
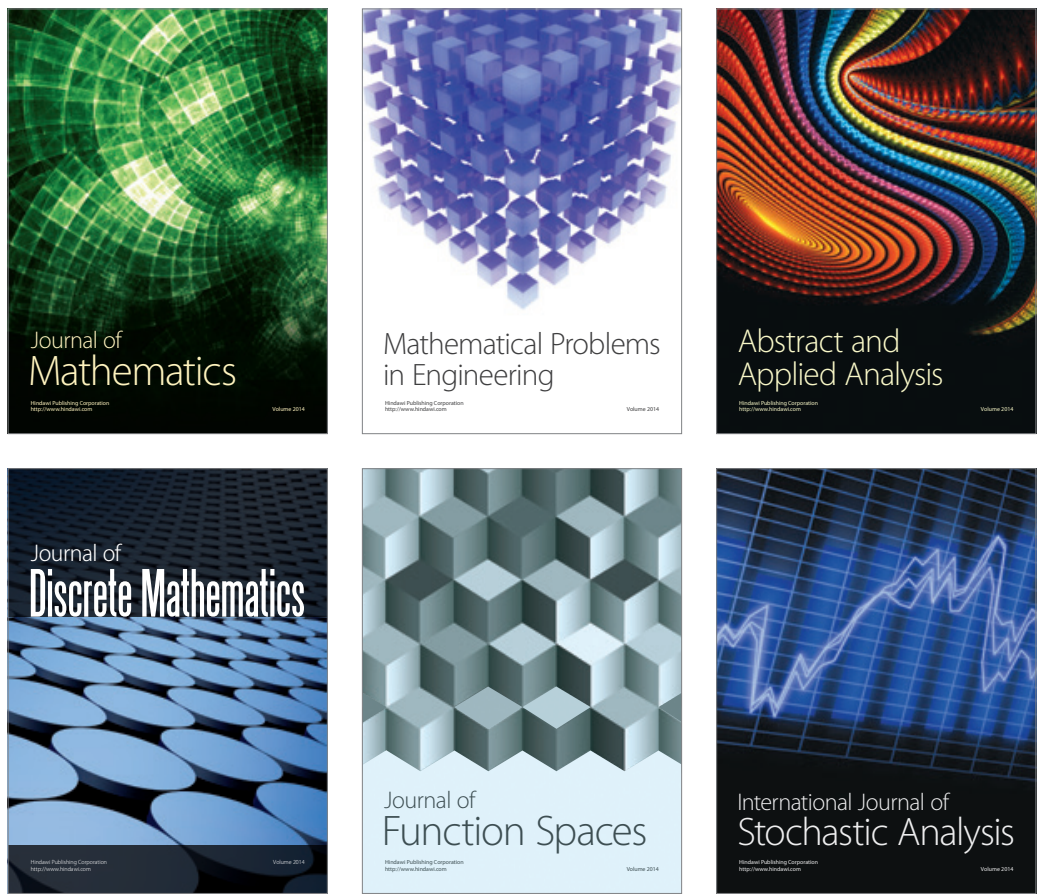

Journal of

Function Spaces

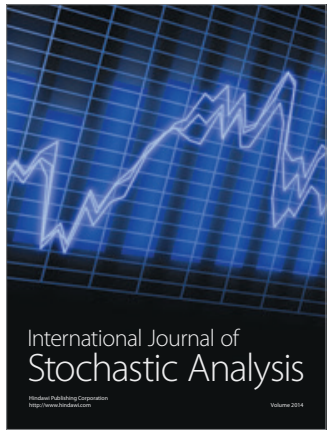

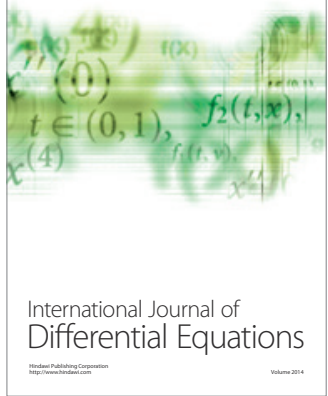
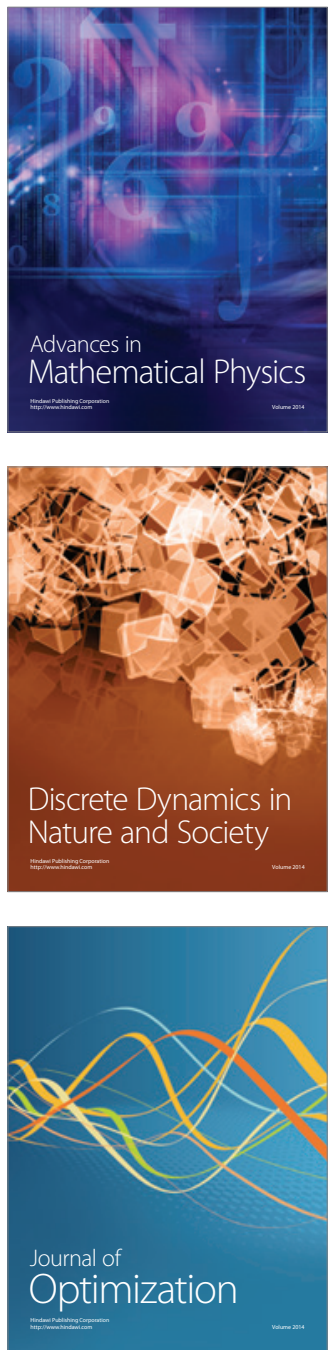